TAIWANESE JOURNAL OF MATHEMATICS

Vol. 19, No. 1, pp. 335-348, February 2015

DOI: $10.11650 /$ tjm.19.2015.4497

This paper is available online at http://journal.taiwanmathsoc.org.tw

\title{
WEIGHTED LIPSCHITZ ESTIMATES FOR COMMUTATORS ON WEIGHTED MORREY-HERZ SPACES
}

\author{
Yan Lin*, Zongguang Liu and $\mathrm{Na} \mathrm{Xu}$
}

\begin{abstract}
In this paper, the authors establish the boundedness of commutators generated by Calderón-Zygmund singular integral operators and weighted Lipschitz functions on weighted Morrey-Herz spaces.
\end{abstract}

\section{INTRODUCTION}

The standard singular integral operator is defined by

$$
T f(x)=\text { p.v. } \int_{\mathbb{R}^{n}} K(x-y) f(y) d y .
$$

A well-known result of Stein in [13] states that if $T$ is bounded on $L^{q}\left(\mathbb{R}^{n}\right), 1<q<\infty$, and

$$
|K(x)| \leq \frac{C}{|x|^{n}}, \quad \forall x \neq 0,
$$

then $T$ is also bounded on the weighted spaces $L_{|x|^{\beta}}^{q}\left(\mathbb{R}^{n}\right),-n<\beta<n(q-1)$, where the range of $\beta$ is the best.

In 1994, the above Stein's result was developed by Soria and Weiss [12] in the following way. The singular integral operator satisfying (1.1) will be replaced by any sublinear operator $T$ satisfying the following size condition: For any $f \in L^{1}\left(\mathbb{R}^{n}\right)$ with compact support and for $x \notin \operatorname{supp} f$,

$$
|T f(x)| \leq C \int_{\mathbb{R}^{n}} \frac{|f(y)|}{|x-y|^{n}} d y .
$$

Received March 5, 2014, accepted May 30, 2014.

Communicated by Yong-Sheng Han.

2010 Mathematics Subject Classification: 42B20, 42B35.

Key words and phrases: Calderón-Zygmund operator, Commutator, Weighted Lipschitz function, MorreyHerz space.

This work was supported by the National Natural Science Foundation of China (Nos. 11001266 and 11171345), Beijing Higher Education Young Elite Teacher Project (No. YETP0946), and the Fundamental Research Funds for the Central Universities (No. 2009QS16).

*Corresponding author. 
Originated from the definition of Coifman, Rochberg and Weiss [1], throughout this paper we focus on the Calderón-Zygmund singular integral operator,

$$
T f(x)=\text { p.v. } \int_{\mathbb{R}^{n}} K(x-y) f(y) d y,
$$

which satisfies

(1) $|K(x)| \leq C|x|^{-n}, x \neq 0$

(2) $|K(x-y)-K(x)| \leq C \frac{|y|}{|x|^{n+1}}, 2|y| \leq|x|$;

(3) $T$ can be extended into a continuous operator on $L^{2}\left(\mathbb{R}^{n}\right)$.

Let $b$ be a locally integrable function on $\mathbb{R}^{n}$ and let $T$ be a Calderón-Zygmund singular integral operator. The commutator $[b, T]$ generated by $b$ and $T$ is defined by

$$
[b, T] f(x)=b T(f)(x)-T(b f)(x) .
$$

Janson [4] proved that $[b, T]$ is bounded on $L^{p}$ for $1<p<\infty$ if and only if $b \in B M O$; see also [1]. Paluszyński [11] showed that $b \in \operatorname{Lip}_{\beta}$ if and only if the commutator $[b, T]$ is bounded from $L^{p}$ to $L^{q}$, where $1<p<q<\infty, 0<\beta<1$ and $1 / q=1 / p-\beta / n$. Lu and Yang [10] obtained the boundedness of commutators generated by singular integrals and Lipschitz functions on Herz spaces. And Lin [6] proved the boundedness of commutators generated by strongly singular Calderón-Zygmund operators and Lipschitz functions on Morrey type spaces.

Let $B_{k}=\left\{x \in \mathbb{R}^{n}:|x| \leq 2^{k}\right\}, E_{k}=B_{k} \backslash B_{k-1}$ and $\chi_{k}=\chi_{E_{k}}$ for $k \in \mathbb{Z}$, where by $\chi_{E}$ we denote the characteristic function of a set $E$. Let $f_{B}=\frac{1}{|B|} \int_{B} f(x) d x$.

Definition 1.1. Let $\alpha \in \mathbb{R}$ and $0<p, q \leq \infty$. The homogeneous Herz space $\dot{K}_{q}^{\alpha, p}\left(\mathbb{R}^{n}\right)$ is defined by

$$
\dot{K}_{q}^{\alpha, p}\left(\mathbb{R}^{n}\right)=\left\{f \in L_{\mathrm{loc}}^{q}\left(\mathbb{R}^{n} \backslash\{0\}\right):\|f\|_{\dot{K}_{q}^{\alpha, p}\left(\mathbb{R}^{n}\right)}<\infty\right\},
$$

where

$$
\|f\|_{\dot{K}_{q}^{\alpha, p}\left(\mathbb{R}^{n}\right)}=\left(\sum_{k=-\infty}^{\infty} 2^{k \alpha p}\left\|f \chi_{k}\right\|_{L^{q}\left(\mathbb{R}^{n}\right)}^{p}\right)^{1 / p}
$$

with usual modifications made when $p=\infty$.

Definition 1.2. Let $1 \leq p \leq q<\infty$. The Morrey space $M_{p}^{q}\left(\mathbb{R}^{n}\right)$ is defined by

$$
M_{p}^{q}\left(\mathbb{R}^{n}\right)=\left\{f \in L_{\mathrm{loc}}^{q}\left(\mathbb{R}^{n}\right):\|f\|_{M_{p}^{q}\left(\mathbb{R}^{n}\right)}<\infty\right\}
$$

where

$$
\|f\|_{M_{p}^{q}\left(\mathbb{R}^{n}\right)}=\sup _{B \subset \mathbb{R}^{n}}|B|^{\frac{1}{q}-\frac{1}{p}}\left(\int_{B}|f(x)|^{p} d x\right)^{1 / p}
$$


with usual modifications made when $p=\infty$.

Definition 1.3. Let $0 \leq \lambda<\infty, \alpha \in \mathbb{R}$ and $0<p, q \leq \infty$. A function $f$ is said to belong to the Morrey-Herz space $M K_{p, q}^{\alpha, \lambda}\left(\mathbb{R}^{n}\right)$ provided that

$$
\|f\|_{M K_{p, q}^{\alpha, \lambda}\left(\mathbb{R}^{n}\right)}=\sup _{k_{0} \in \mathbb{Z}} 2^{-k_{0} \lambda}\left(\sum_{k=-\infty}^{k_{0}} 2^{k \alpha p}\left\|f \chi_{k}\right\|_{L^{q}\left(\mathbb{R}^{n}\right)}^{p}\right)^{1 / p}<\infty
$$

with usual modifications made when $p=\infty$.

A non-negative function $\mu$ defined on $\mathbb{R}^{n}$ is called a weight if it is locally integrable. A weight $\mu$ is said to belong to the Muckenhoupt class $A_{p}\left(\mathbb{R}^{n}\right)$ for $1<p<\infty$, if there exists a constant $C>0$ such that

$$
\left(\frac{1}{|B|} \int_{B} \mu(x) d x\right)\left(\frac{1}{|B|} \int_{B} \mu(x)^{-\frac{1}{p-1}} d x\right)^{p-1} d x \leq C,
$$

for every ball $B \subset \mathbb{R}^{n}$. The class $A_{1}\left(\mathbb{R}^{n}\right)$ is defined replacing the above inequality by

$$
\frac{1}{|B|} \int_{B} \mu(x) d x \leq C \mu(x), \quad \text { a.e. } x \in \mathbb{R}^{n} .
$$

A function $\mu \in A_{\infty}$ if it satisfies the condition of $A_{p}$ for some $p>1$. It is well-known that if $1<p<q<\infty$, then we have $A_{1} \subset A_{p} \subset A_{q}$.

Definition 1.4. Let $0 \leq \lambda<\infty, \alpha \in \mathbb{R}$ and $0<p, q \leq \infty$, and let $\mu_{1}$ and $\mu_{2}$ be non-negative weighted functions. A function $f$ is said to belong to the weighted Morrey-Herz space $M K_{p, q}^{\alpha, \lambda}\left(\mu_{1}, \mu_{2}\right)$ provided that

$$
\|f\|_{M K_{p, q}^{\alpha, \lambda}\left(\mu_{1}, \mu_{2}\right)}=\sup _{k_{0} \in \mathbb{Z}} 2^{-k_{0} \lambda}\left(\sum_{k=-\infty}^{k_{0}} \mu_{1}\left(B_{k}\right)^{\alpha p / n}\left\|f \chi_{k}\right\|_{L^{q}\left(\mu_{2}\right)}^{p}\right)^{1 / p}<\infty .
$$

It is easy to see that $\dot{K}_{q}^{0, q}\left(\mathbb{R}^{n}\right)=L^{q}\left(\mathbb{R}^{n}\right)$ for $0<q \leq \infty, M_{p}^{p}\left(\mathbb{R}^{n}\right)=L^{p}\left(\mathbb{R}^{n}\right)$ with $1 \leq p<\infty$, so Herz spaces and Morrey spaces are the generalization of Lebesgue spaces. Meanwhile $M K_{p, q}^{\alpha, 0}\left(\mathbb{R}^{n}\right)=\dot{K}_{q}^{\alpha, p}\left(\mathbb{R}^{n}\right)$, and $M K_{q, q}^{0, \lambda}\left(\mathbb{R}^{n}\right) \supseteq M_{q}^{\frac{n q}{n-\lambda q}}\left(\mathbb{R}^{n}\right)$, where $1 \leq q<\infty, 0 \leq \lambda<n / q$, so the special cases of Morrey-Herz spaces are Morrey spaces and Herz spaces. Obviously, when $\mu_{1}=\mu_{2} \equiv 1, M K_{p, q}^{\alpha, \lambda}\left(\mu_{1}, \mu_{2}\right)=$ $M K_{p, q}^{\alpha, \lambda}\left(\mathbb{R}^{n}\right)$.

Recently the necessary and sufficient conditions for boundedness of some commutators of singular integrals with weighted Lipschitz functions on weighted Lebesgue spaces are established by $\mathrm{Hu}$ and $\mathrm{Gu}$ in [3]. After this, the boundedness of commutators generated by weighted Lipschitz functions and singular integrals or fractional 
integrals with rough kernels on weighted Lebesgue spaces was established in [7,8]. And the boundedness of commutators generated by singular integrals and weighted Lipschitz functions on weighted Herz spaces has been obtained in [9]. Since MorreyHerz spaces are generalizations of Herz spaces, a natural question is whether this kind of commutators also have boundedness on weighted Morrey-Herz spaces. The answer is affirmative. The main purpose of this paper is to generalize the above results and establish the corresponding boundedness on weighted Morrey-Herz spaces.

\section{MAIN Results}

In order to obtain our main results, first we need introduce some necessary notations and requisite lemmas.

Definition 2.1. [2]. We say that a locally integrable function $f$ belongs to the weighted Lipschitz space $\operatorname{Lip}_{\beta, \mu}^{p}$ for $1 \leq p \leq \infty, 0<\beta<1$ and $\mu \in A_{\infty}$, if

$$
\sup _{B} \frac{1}{\mu(B)^{\beta / n}}\left[\frac{1}{\mu(B)} \int_{B}\left|f(x)-f_{B}\right|^{p} \mu(x)^{1-p} d x\right]^{1 / p}<\infty,
$$

where $B$ is any ball in $\mathbb{R}^{n}$.

Modulo constants, the Banach space of such functions is denoted by $\operatorname{Lip}_{\beta, \mu}^{p}$. The smallest bound $C$ satisfying conditions above is taken to be the norm of $f$ in this space, and is denoted by $\|f\|_{L i p_{\beta, \mu}^{p}}$. Put $\operatorname{Lip}_{\beta, \mu}=\operatorname{Lip}_{\beta, \mu}^{1}$. Obviously, for the case $\mu \equiv 1$, the space $\operatorname{Lip}_{\beta, \mu}$ is the classical Lipschitz space $\operatorname{Lip}_{\beta}$. Thus, weighted Lipschitz spaces are generalizations of classical Lipschitz spaces.

If $\mu \in A_{1}\left(\mathbb{R}^{n}\right)$, García-Cuerva in [2] proved that the space $\operatorname{Lip}_{\beta, \mu}^{p}$ coincide, and the norm of $\|\cdot\|_{L i p_{\beta, \mu}^{p}}$ are equivalent with respect to different values of $p$ provided that $1 \leq p \leq \infty$. That is $\|f\|_{L i p_{\beta, \mu}^{p}} \sim\|f\|_{L_{i p_{\beta, \mu}}}$, where $1 \leq p \leq \infty$.

Lemma 2.1. [5]. Let $\mu \in A_{1}$, then there are constants $C_{1}, C_{2}$ and $0<\delta<1$ depending only on $A_{1}$-constant of $\mu$, such that for any measurable subset $E$ of a ball $B$,

$$
C_{1} \frac{|E|}{|B|} \leq \frac{\mu(E)}{\mu(B)} \leq C_{2}\left(\frac{|E|}{|B|}\right)^{\delta} .
$$

Lemma 2.2. [9]. Let $\mu \in A_{1}$ and $b \in \operatorname{Lip}_{\beta, \mu}$, then there is a constant $C$ such that for all $j, k \in \mathbb{Z}$ with $j>k$,

$$
\left|b_{B_{j}}-b_{B_{k}}\right| \leq C(j-k)\|b\|_{L i p_{\beta, \mu}} \mu\left(B_{j}\right)^{\frac{\beta}{n}} \frac{\mu\left(B_{k}\right)}{\left|B_{k}\right|} .
$$


Lemma 2.3. [9]. Let $\mu \in A_{1}$, then there is a constant $C$ such that for $1<p<\infty$ and any ball $B$,

$$
\int_{B} \mu(x)^{1-p^{\prime}} d x \leq C|B|^{p^{\prime}} \mu(B)^{1-p^{\prime}}
$$

where $1 / p+1 / p^{\prime}=1$.

Recently, the authors of [3] discussed the boundedness of commutators generated by singular integrals and weighted Lipschitz functions on weighted Lebesgue spaces.

Theorem A. [3]. Let $T$ be a Calderón-Zygmund singular integral operator. Let $\mu \in A_{1}, 1 / q=1 / p-\beta / n$ for $0<\beta<1$ and $1<p<q<\infty$. Let $b \in$ Lip $_{\beta, \mu}$. Then the commutator $[b, T]$ is bounded from $L^{p}(\mu)$ to $L^{q}\left(\mu^{1-q}\right)$.

The purpose of this paper is to state the boundedness of commutators generated by singular integrals and weighted Lipschitz functions on weighted Morrey-Herz spaces. The main result is as follows:

Theorem 2.1. Let $T$ be a Calderón-Zygmund singular integral operator and $\delta$ be defined as in Lemma 2.1. Let $b \in \operatorname{Lip}_{\beta, \mu}, \mu \in A_{1}, 0<\beta<1$, then the commutator $[b, T]$ is bounded from $M K_{p, q_{1}}^{\alpha, \lambda}(\mu, \mu)$ to $M K_{p, q_{2}}^{\alpha, \lambda}\left(\mu, \mu^{1-q_{2}}\right)$, where $0 \leq \lambda<\infty$, $0<p \leq \infty, 1<q_{1}, q_{2}<\infty, 1 / q_{2}=1 / q_{1}-\beta / n, 1 / \widetilde{q}_{1}=1-\min \left\{1-\frac{1}{\delta q_{1}}, \delta-\frac{1}{q_{1}}\right\}$, $1 / \widetilde{q}_{2}=\min \left\{\frac{1}{q_{1}}-\frac{\beta}{n \delta}, \frac{\delta}{q_{1}}-\frac{\beta}{n}\right\}$, and $\lambda / \delta-n / \widetilde{q}_{2}<\alpha<n\left(1-1 / \widetilde{q}_{1}\right)$.

Note that if $\delta=1$, then $\widetilde{q}_{1}=q_{1}$ and $\widetilde{q}_{2}=q_{2}$, and hence when $\mu \equiv 1$, we have $\operatorname{Lip}_{\beta, \mu}\left(\mathbb{R}^{n}\right)=\operatorname{Lip}_{\beta}\left(\mathbb{R}^{n}\right), M K_{p, q_{1}}^{\alpha, \lambda}(\mu, \mu)=M K_{p, q_{1}}^{\alpha, \lambda}\left(\mathbb{R}^{n}\right)$, and $M K_{p, q_{2}}^{\alpha, \lambda}\left(\mu, \mu^{1-q_{2}}\right)=$ $M K_{p, q_{2}}^{\alpha, \lambda}\left(\mathbb{R}^{n}\right)$. Thus, we can obtain the boundedness of commutators generated by singular integrals and classical Lipschitz functions on Morrey-Herz spaces as a corollary of Theorem 2.1.

Corollary 2.1. Let $T$ be a Calderon-Zygmund singular integral operator. Let $b \in \operatorname{Lip}_{\beta}\left(\mathbb{R}^{n}\right), 0<\beta<1$, then the commutator $[b, T]$ is bounded from $M K_{p, q_{1}}^{\alpha, \lambda}\left(\mathbb{R}^{n}\right)$ to $M K_{p, q_{2}}^{\alpha, \lambda}\left(\mathbb{R}^{n}\right)$, where $0 \leq \lambda<\infty, 0<p \leq \infty, 1<q_{1}, q_{2}<\infty, 1 / q_{2}=1 / q_{1}-\beta / n$, and $\lambda-n / q_{2}<\alpha<n\left(1-1 / q_{1}\right)$.

When $\lambda=0$, the weighted Morrey-Herz space $M K_{p, q}^{\alpha, 0}\left(\mu_{1}, \mu_{2}\right)$ is the homogeneous weighted Herz space $\dot{K}_{q}^{\alpha, p}\left(\mu_{1}, \mu_{2}\right)$. Thus, we can obtain the boundedness of commutators generated by singular integrals and weighted Lipschitz functions on weighted Herz spaces as another corollary of Theorem 2.1.

Corollary 2.2. Let $T$ be a Calderón-Zygmund singular integral operator and $\delta$ be defined as in Lemma 2.1. Let $b \in$ Lip $_{\beta, \mu}, \mu \in A_{1}, 0<\beta<1$, then the commutator $[b, T]$ is bounded from $\dot{K}_{q_{1}}^{\alpha, p}(\mu, \mu)$ to $\dot{K}_{q_{2}}^{\alpha, p}\left(\mu, \mu^{1-q_{2}}\right)$, where $0<p \leq \infty, 1<q_{1}, q_{2}<$ 
$\infty, 1 / q_{2}=1 / q_{1}-\beta / n, 1 / \widetilde{q}_{1}=1-\min \left\{1-\frac{1}{\delta q_{1}}, \delta-\frac{1}{q_{1}}\right\}, 1 / \widetilde{q}_{2}=\min \left\{\frac{1}{q_{1}}-\frac{\beta}{n \delta}, \frac{\delta}{q_{1}}-\frac{\beta}{n}\right\}$, and $-n / \widetilde{q}_{2}<\alpha<n\left(1-1 / \widetilde{q}_{1}\right)$.

Remark 2.1. Actually, the result of Corollary 2.2 has been obtained in [9], however there are some calculation errors in the proof of Theorem 2.5 in [9]. The exact range of $\alpha$ there should be $-n / \widetilde{q}_{2}<\alpha<n\left(1-1 / \widetilde{q}_{1}\right)$.

\section{Proof of Theorem 2.1}

In this section, we will give the proof of Theorem 2.1.

Proof. We only consider the case $0<p<\infty$ and omit the details of the case $p=\infty$ since their similarity. Set

$$
f=\sum_{j=-\infty}^{\infty} f \chi_{j}:=\sum_{j=-\infty}^{\infty} f_{j} .
$$

Then, by the Minkowski inequality, we write

$$
\begin{aligned}
& \quad\|[b, T] f\|_{M K_{p, q_{2}}^{\alpha, \lambda}\left(\mu, \mu^{1-q_{2}}\right)} \\
& =\sup _{k_{0} \in \mathbb{Z}} 2^{-k_{0} \lambda}\left(\sum_{k=-\infty}^{k_{0}} \mu\left(B_{k}\right)^{\alpha p / n}\left\|([b, T] f) \chi_{k}\right\|_{L^{q_{2}}\left(\mu^{1-q_{2}}\right)}^{p}\right)^{\frac{1}{p}} \\
& \left.=\sup _{k_{0} \in \mathbb{Z}} 2^{-k_{0} \lambda}\left(\sum_{k=-\infty}^{k_{0}} \mu\left(B_{k}\right)^{\alpha p / n}\left\|\sum_{j=-\infty}^{\infty}\left([b, T] f \chi_{j}\right) \chi_{k}\right\|_{L^{q_{2}\left(\mu^{1-q_{2}}\right)}}^{p}\right)^{\frac{1}{p}}\right)^{\frac{1}{p}} \\
& \leq C \sup _{k_{0} \in \mathbb{Z}} 2^{-k_{0} \lambda}\left(\sum_{k=-\infty}^{k_{0}} \mu\left(B_{k}\right)^{\alpha p / n}\left(\sum_{j=-\infty}^{k-2}\left\|\left([b, T] f \chi_{j}\right) \chi_{k}\right\|_{L^{q_{2}}\left(\mu^{1-q_{2}}\right)}\right)^{p}\right. \\
& \quad+C \sup _{k_{0} \in \mathbb{Z}} 2^{-k_{0} \lambda}\left(\sum_{k=-\infty}^{k_{0}} \mu\left(B_{k}\right)^{\alpha p / n}\left(\sum_{j=k-1}^{k+1}\left\|\left([b, T] f \chi_{j}\right) \chi_{k}\right\|_{L^{q_{2}}\left(\mu^{1-q_{2}}\right)}\right)^{\frac{1}{p}}\right)^{p} \\
& \quad+C \sup _{k_{0} \in \mathbb{Z}} 2^{-k_{0} \lambda}\left(\sum_{k=-\infty}^{k_{0}} \mu\left(B_{k}\right)^{\alpha p / n}\left(\sum_{j=k+2}^{\infty}\left\|\left([b, T] f \chi_{j}\right) \chi_{k}\right\|_{L^{q_{2}}\left(\mu^{1-q_{2}}\right)}\right)^{\frac{1}{p}}\right. \\
& :=I_{1}+I_{2}+I_{3} .
\end{aligned}
$$

For $I_{2}$, by Theorem A and Lemma 2.1, we find

$$
\begin{aligned}
I_{2} & \leq C \sup _{k_{0} \in \mathbb{Z}} 2^{-k_{0} \lambda}\left(\sum_{k=-\infty}^{k_{0}} \mu\left(B_{k}\right)^{\alpha p / n}\left(\sum_{j=k-1}^{k+1}\left\|[b, T]\left(f \chi_{j}\right)\right\|_{L^{q_{2}}\left(\mu^{1-q_{2}}\right)}\right)^{p}\right)^{\frac{1}{p}} \\
& \leq C\|b\|_{L i p_{\beta}, \mu} \sup _{k_{0} \in \mathbb{Z}} 2^{-k_{0} \lambda}\left(\sum_{k=-\infty}^{k_{0}} \mu\left(B_{k}\right)^{\alpha p / n}\left(\sum_{j=k-1}^{k+1}\left\|f \chi_{j}\right\|_{L^{q_{1}}(\mu)}\right)^{p}\right)^{\frac{1}{p}}
\end{aligned}
$$




$$
\begin{aligned}
& \leq C\|b\|_{L i p_{\beta}, \mu} \sup _{k_{0} \in \mathbb{Z}} 2^{-k_{0} \lambda}\left(\sum_{k=-\infty}^{k_{0}} \mu\left(B_{k}\right)^{\alpha p / n}\left\|f \chi_{k}\right\|_{L^{q_{1}}(\mu)}^{p}\right)^{\frac{1}{p}} \\
& =C\|b\|_{L i p_{\beta}, \mu}\|f\|_{M K_{p, q_{1}}^{\alpha, \lambda}(\mu, \mu)^{.}}
\end{aligned}
$$

To obtain the estimates for $I_{1}$ and $I_{3}$, we first observe that for $j, k \in \mathbb{Z}$ with $|j-k| \geq 2$,

$$
\left([b, T] f \chi_{j}\right)(x)=\left(b(x)-b_{B_{j}}\right) T\left(f \chi_{j}\right)(x)-T\left(\left(b-b_{B_{j}}\right) f \chi_{j}\right)(x) .
$$

Thus,

$$
\begin{aligned}
\| & \left([b, T] f \chi_{j}\right) \chi_{k} \|_{L^{q_{2}}\left(\mu^{1-q_{2}}\right)} \\
= & \left(\int_{E_{k}}\left|[b, T]\left(f \chi_{j}\right)(x)\right|^{q_{2}} \mu(x)^{1-q_{2}} d x\right)^{\frac{1}{q_{2}}} \\
= & \left(\int_{E_{k}}\left|\left(b(x)-b_{B_{j}}\right) T\left(f \chi_{j}\right)(x)-T\left(\left(b-b_{B_{j}}\right) f \chi_{j}\right)(x)\right|^{q_{2}} \mu(x)^{1-q_{2}} d x\right)^{\frac{1}{q_{2}}} \\
\leq & \left(\int_{E_{k}}\left|b(x)-b_{B_{j}}\right|^{q_{2}}\left|T\left(f \chi_{j}\right)(x)\right|^{q_{2}} \mu(x)^{1-q_{2}} d x\right)^{\frac{1}{q_{2}}} \\
& +\left(\int_{E_{k}}\left|T\left(\left(b-b_{B_{j}}\right) f \chi_{j}\right)(x)\right|^{q_{2}} \mu(x)^{1-q_{2}} d x\right)^{\frac{1}{q_{2}}} \\
= & \left(\int_{E_{k}}\left|b(x)-b_{B_{j}}\right|^{q_{2}}\left|\int_{E_{j}} K(x-y)\left(f \chi_{j}\right)(y) d y\right|^{q_{2}} \mu(x)^{1-q_{2}} d x\right)^{\frac{1}{q_{2}}} \\
& +\left(\int_{E_{k}}\left|\int_{E_{j}} K(x-y)\left(b(y)-b_{B_{j}}\right)\left(f \chi_{j}\right)(y) d y\right|^{q_{2}} \mu(x)^{1-q_{2}} d x\right)^{\frac{1}{q_{2}}} \\
\leq & \left(\int_{E_{k}}\left|b(x)-b_{B_{j}}\right|^{q_{2}}\left(\int_{E_{j}} \frac{1}{|x-y|^{n}}\left|\left(f \chi_{j}\right)(y)\right| d y\right)^{q_{2}} \mu(x)^{1-q_{2}} d x\right)^{\frac{1}{q_{2}}} \\
& +\left(\int_{E_{k}}\left(\int_{E_{j}} \frac{1}{|x-y|^{n}}\left|b(y)-b_{B_{j}}\right|\left|\left(f \chi_{j}\right)(y)\right| d y\right)^{q_{2}} \mu(x)^{1-q_{2}} d x\right)^{\frac{1}{q_{2}}} \\
:= & D_{1}(j, k)+D_{2}(j, k) .
\end{aligned}
$$

Now, let us estimate $I_{1}$. If $j \leq k-2$, then

$$
D_{1}(j, k) \leq C 2^{-k n}\left(\int_{E_{k}}\left|b(x)-b_{B_{j}}\right|^{q_{2}} \mu(x)^{1-q_{2}} d x\right)^{\frac{1}{q_{2}}}\left(\int_{E_{j}}\left|\left(f \chi_{j}\right)(y)\right| d y\right),
$$

and

$$
D_{2}(j, k) \leq C 2^{-k n}\left(\int_{E_{k}} \mu(x)^{1-q_{2}} d x\right)^{\frac{1}{q_{2}}}\left(\int_{E_{j}}\left|b(y)-b_{B_{j}}\right|\left|\left(f \chi_{j}\right)(y)\right| d y\right) .
$$


By Lemma 2.2, Lemma 2.3 and Lemma 2.1, for $j \leq k-2$ we have

$$
\begin{aligned}
& \left(\int_{E_{k}}\left|b(x)-b_{B_{j}}\right|^{q_{2}} \mu(x)^{1-q_{2}} d x\right)^{\frac{1}{q_{2}}} \\
\leq & \left(\int_{B_{k}}\left|b(x)-b_{B_{k}}\right|^{q_{2}} \mu(x)^{1-q_{2}} d x\right)^{\frac{1}{q_{2}}}+\left(\int_{B_{k}}\left|b_{B_{k}}-b_{B_{j}}\right|^{q_{2}} \mu(x)^{1-q_{2}} d x\right)^{\frac{1}{q_{2}}} \\
\leq & C\|b\|_{L i p_{\beta, \mu}} \mu\left(B_{k}\right)^{\frac{\beta}{n}} \mu\left(B_{k}\right)^{\frac{1}{q_{2}}}+\left|b_{B_{k}}-b_{B_{j}}\right|\left(\int_{B_{k}} \mu(x)^{1-q_{2}} d x\right)^{\frac{1}{q_{2}}} \\
\leq & C\|b\|_{L i p_{\beta, \mu}} \mu\left(B_{k}\right)^{\frac{\beta}{n}} \mu\left(B_{k}\right)^{\frac{1}{q_{2}}}+C(k-j)\|b\|_{L i p_{\beta, \mu}} \mu\left(B_{k}\right)^{\frac{\beta}{n}} \frac{\mu\left(B_{j}\right)}{\left|B_{j}\right|}\left|B_{k}\right| \mu\left(B_{k}\right)^{\frac{1-q_{2}}{q_{2}}} \\
= & C\|b\|_{L i p_{\beta, \mu}} \mu\left(B_{k}\right)^{\frac{1}{q_{1}}}+C\|b\|_{L i p_{\beta, \mu}}(k-j) \mu\left(B_{k}\right)^{\frac{1}{q_{1}}} \frac{\left|B_{k}\right|}{\left|B_{j}\right|} \frac{\mu\left(B_{j}\right)}{\mu\left(B_{k}\right)} \\
\leq & C\|b\|_{L i p_{\beta, \mu}} \mu\left(B_{k}\right)^{\frac{1}{q_{1}}}+C\|b\|_{L i p_{\beta, \mu}}(k-j) \mu\left(B_{k}\right)^{\frac{1}{q_{1}}} \frac{\left|B_{k}\right|}{\left|B_{j}\right|}\left(\frac{\left|B_{j}\right|}{\left|B_{k}\right|}\right)^{\delta} \\
\leq & C\|b\|_{L i p_{\beta, \mu}}(k-j) \mu\left(B_{k}\right)^{\frac{1}{q_{1}}} 2^{(k-j) n(1-\delta) .}
\end{aligned}
$$

By Hölder's inequality and Lemma 2.3 , denoting that $1 / q_{1}+1 / q_{1}^{\prime}=1$, for all $j \in \mathbb{Z}$, we obtain

$$
\begin{aligned}
\int_{E_{j}}\left|\left(f \chi_{j}\right)(y)\right| d y & \leq\left(\int_{B_{j}}\left|\left(f \chi_{j}\right)(y)\right|^{q_{1}} \mu(y) d y\right)^{\frac{1}{q_{1}}}\left(\int_{B_{j}} \mu(y)^{1-q_{1}^{\prime}} d y\right)^{\frac{1}{q_{1}^{\prime}}} \\
& \leq C\left\|f \chi_{j}\right\|_{L^{q_{1}(\mu)}}\left|B_{j}\right| \mu\left(B_{j}\right)^{-\frac{1}{q_{1}}} \\
& =C 2^{j n}\left\|f \chi_{j}\right\|_{L^{q_{1}}(\mu)} \mu\left(B_{j}\right)^{-\frac{1}{q_{1}}}
\end{aligned}
$$

By the above two estimates and Lemma 2.1, for $j \leq k-2$ we have

$$
\begin{aligned}
& D_{1}(j, k) \leq C\|b\|_{L i p_{\beta, \mu}} 2^{-k n}(k-j) \mu\left(B_{k}\right)^{\frac{1}{q_{1}}} 2^{(k-j) n(1-\delta)} 2^{j n}\left\|f \chi_{j}\right\|_{L^{q_{1}}(\mu)} \mu\left(B_{j}\right)^{-\frac{1}{q_{1}}} \\
& =C\|b\|_{L i p_{\beta, \mu}}(k-j) 2^{-(k-j) n \delta}\left(\frac{\mu\left(B_{k}\right)}{\mu\left(B_{j}\right)}\right)^{\frac{1}{q_{1}}}\left\|f \chi_{j}\right\|_{L^{q_{1}}(\mu)} \\
& \leq C\|b\|_{L i p_{\beta, \mu}}(k-j) 2^{-(k-j) n \delta}\left(\frac{\left|B_{k}\right|}{\left|B_{j}\right|}\right)^{\frac{1}{q_{1}}}\left\|f \chi_{j}\right\|_{L^{q_{1}}(\mu)} \\
& =C\|b\|_{L i p_{\beta, \mu}}(k-j) 2^{(k-j) n\left(\frac{1}{q_{1}}-\delta\right)}\left\|f \chi_{j}\right\|_{L^{q_{1}}(\mu)} .
\end{aligned}
$$

By Hölder's inequality, Lemma 2.3 and Lemma 2.1, for $j \leq k-2$ we have 


$$
\begin{aligned}
& D_{2}(j, k) \leq C 2^{-k n}\left|B_{k}\right| \mu\left(B_{k}\right)^{\frac{1}{q_{2}}-1}\left(\int_{B_{j}}\left|b(y)-b_{B_{j}}\right|^{q_{1}^{\prime}} \mu(y)^{\frac{1}{-q_{1}} q_{1}^{\prime}} d y\right)^{\frac{1}{q_{1}^{\prime}}} \\
& \times\left(\int_{B_{j}}\left|\left(f \chi_{j}\right)(y)\right|^{q_{1}} \mu(y) d y\right)^{\frac{1}{q_{1}}} \\
& \leq C\|b\|_{L i p_{\beta, \mu}} \mu\left(B_{k}\right)^{\frac{1}{q_{2}}-1} \mu\left(B_{j}\right)^{\frac{1}{q_{1}^{\prime}}} \mu\left(B_{j}\right)^{\frac{\beta}{n}}\left\|f \chi_{j}\right\|_{L^{q_{1}}(\mu)}
\end{aligned}
$$

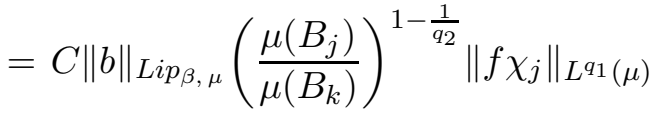

$$
\begin{aligned}
& \leq C\|b\|_{L i p_{\beta, \mu}}\left(\frac{\left|B_{j}\right|}{\left|B_{k}\right|}\right)^{\delta\left(1-\frac{1}{q_{2}}\right)}\left\|f \chi_{j}\right\|_{L^{q_{1}}(\mu)} \\
& =C\|b\|_{L i p_{\beta, \mu}} 2^{(j-k) n \delta\left(1-\frac{1}{q_{2}}\right)}\left\|f \chi_{j}\right\|_{L^{q_{1}}(\mu)} .
\end{aligned}
$$

Combining the estimates for $D_{1}(j, k)$ and $D_{2}(j, k)$, we obtain that if $j \leq k-2$, then

$$
\begin{aligned}
& \left\|\left([b, T] f \chi_{j}\right) \chi_{k}\right\|_{L^{q_{2}}\left(\mu^{1-q_{2}}\right)} \\
\leq & C\|b\|_{L_{p_{\beta, \mu}}}(k-j) 2^{(k-j) n\left(\frac{1}{q_{1}}-\delta\right)}\left\|f \chi_{j}\right\|_{L^{q_{1}}(\mu)} \\
& +C\|b\|_{L i p_{\beta, \mu}} 2^{(k-j) n \delta\left(\frac{1}{q_{2}}-1\right)}\left\|f \chi_{j}\right\|_{L^{q_{1}}(\mu)} \\
\leq & C\|b\|_{L i p_{\beta, \mu}}(k-j) 2^{(k-j) n\left(\frac{1}{q_{1}}-\delta\right)}\left\|f \chi_{j}\right\|_{L^{q_{1}}(\mu)} .
\end{aligned}
$$

So we have

$$
\begin{aligned}
I_{1} \leq & C \sup _{k_{0} \in \mathbb{Z}} 2^{-k_{0} \lambda}\left(\sum_{k=-\infty}^{k_{0}} \mu\left(B_{k}\right)^{\alpha p / n}\right. \\
& \left.\times\left(\sum_{j=-\infty}^{k-2}\|b\|_{L i p_{\beta, \mu}}(k-j) 2^{(k-j) n\left(\frac{1}{q_{1}}-\delta\right)}\left\|f \chi_{j}\right\|_{L^{q_{1}}(\mu)}\right)^{p}\right)^{\frac{1}{p}} \\
= & C\|b\|_{L i p_{\beta, \mu}} \sup _{k_{0} \in \mathbb{Z}} 2^{-k_{0} \lambda}\left(\sum _ { k = - \infty } ^ { k _ { 0 } } \left(\sum_{j=-\infty}^{k-2} \mu\left(B_{j}\right)^{\frac{\alpha}{n}}\right.\right. \\
& \left.\left.\times\left(\frac{\mu\left(B_{k}\right)}{\mu\left(B_{j}\right)}\right)^{\frac{\alpha}{n}}(k-j) 2^{(k-j) n\left(\frac{1}{q_{1}}-\delta\right)}\left\|f \chi_{j}\right\|_{L^{q_{1}}(\mu)}\right)^{p}\right)^{\frac{1}{p}} .
\end{aligned}
$$

Let $W(j, k)=(k-j) 2^{(k-j) \delta\left(\alpha-n\left(1-1 / \widetilde{q}_{1}\right)\right)}$ and $M(j, k)=\left(\frac{\mu\left(B_{k}\right)}{\mu\left(B_{j}\right)}\right)^{\frac{\alpha}{n}}(k-j) 2^{(k-j) n\left(\frac{1}{q_{1}}-\delta\right)}$. If $\alpha \geq 0$, then by Lemma 2.1,

$$
\left(\frac{\mu\left(B_{k}\right)}{\mu\left(B_{j}\right)}\right)^{\alpha / n} \leq C\left(\frac{\left|B_{k}\right|}{\left|B_{j}\right|}\right)^{\alpha / n}=C 2^{(k-j) \alpha},
$$


so

$$
\begin{aligned}
M(j, k) & \leq C 2^{(k-j) \alpha}(k-j) 2^{(k-j) n\left(\frac{1}{q_{1}}-\delta\right)} \\
& =C(k-j) 2^{(k-j)\left[\alpha-n\left(\delta-\frac{1}{q_{1}}\right)\right]} \\
& \leq C(k-j) 2^{(k-j)\left[\alpha-n\left(1-\frac{1}{q_{1}}\right)\right]} \\
& \leq C W(j, k) .
\end{aligned}
$$

If $\alpha<0$, then by Lemma 2.1,

$$
\left(\frac{\mu\left(B_{k}\right)}{\mu\left(B_{j}\right)}\right)^{\alpha / n} \leq C\left(\frac{\left|B_{k}\right|}{\left|B_{j}\right|}\right)^{\delta \alpha / n}=C 2^{(k-j) \delta \alpha},
$$

so

$$
\begin{aligned}
M(j, k) & \leq C 2^{(k-j) \delta \alpha}(k-j) 2^{(k-j) n\left(\frac{1}{q_{1}}-\delta\right)} \\
& =C(k-j) 2^{(k-j) \delta\left[\alpha-n\left(1-\frac{1}{\delta q_{1}}\right)\right]} \\
& \leq C W(j, k) .
\end{aligned}
$$

Thus, in both cases $\alpha \geq 0$ and $\alpha<0$, we have

$I_{1} \leq C\|b\|_{L i p_{\beta, \mu},} \sup _{k_{0} \in \mathbb{Z}} 2^{-k_{0} \lambda}\left(\sum_{k=-\infty}^{k_{0}}\left(\sum_{j=-\infty}^{k-2} W(j, k) \mu\left(B_{j}\right)^{\alpha / n}\left\|f \chi_{j}\right\|_{L^{q_{1}}(\mu)}\right)^{p}\right)^{\frac{1}{p}}$.

If $0<p \leq 1$, then it follows from $\alpha<n\left(1-1 / \widetilde{q}_{1}\right)$ that

$$
\begin{aligned}
I_{1} & \leq C\|b\|_{L i p_{\beta, \mu},} \sup _{k_{0} \in \mathbb{Z}} 2^{-k_{0} \lambda}\left(\sum_{k=-\infty}^{k_{0}} \sum_{j=-\infty}^{k-2} W(j, k)^{p} \mu\left(B_{j}\right)^{\frac{\alpha p}{n}}\left\|f \chi_{j}\right\|_{L^{q_{1}(\mu)}}^{p}\right)^{\frac{1}{p}} \\
& \leq C\|b\|_{L i p_{\beta, \mu}} \sup _{k_{0} \in \mathbb{Z}} 2^{-k_{0} \lambda}\left(\sum_{j=-\infty}^{k_{0}-2} \mu\left(B_{j}\right)^{\frac{\alpha p}{n}}\left\|f \chi_{j}\right\|_{L^{q_{1}(\mu)}}^{p}\left(\sum_{k=j+2}^{\infty} W(j, k)^{p}\right)\right)^{\frac{1}{p}} \\
& \leq C\|b\|_{L i p_{\beta, \mu}} \sup _{k_{0} \in \mathbb{Z}} 2^{-k_{0} \lambda}\left(\sum_{j=-\infty}^{k_{0}} \mu\left(B_{j}\right)^{\frac{\alpha p}{n}}\left\|f \chi_{j}\right\|_{L^{q_{1}(\mu)}}^{p}\right)^{\frac{1}{p}} \\
& =C\|b\|_{L i p_{\beta, \mu}, \mu f \|_{M K_{p}, q_{1}}^{\alpha, \lambda}(\mu, \mu)} .
\end{aligned}
$$

When $1<p<\infty$, by Hölder's inequality and $\alpha<n\left(1-1 / \widetilde{q}_{1}\right)$, we have

$$
\begin{aligned}
I_{1} \leq C\|b\|_{L i p_{\beta, \mu}} \sup _{k_{0} \in \mathbb{Z}} 2^{-k_{0} \lambda}\left(\sum_{k=-\infty}^{k_{0}}\left(\sum_{j=-\infty}^{k-2} W(j, k)\right)^{\frac{p}{p^{\prime}}}\right. \\
\left.\times\left(\sum_{j=-\infty}^{k-2} \mu\left(B_{j}\right)^{\frac{\alpha p}{n}}\left\|f \chi_{j}\right\|_{L^{q_{1}(\mu)}}^{p} W(j, k)\right)\right)^{\frac{1}{p}}
\end{aligned}
$$




$$
\begin{aligned}
& =C\|b\|_{L i p_{\beta, \mu}} \sup _{k_{0} \in \mathbb{Z}} 2^{-k_{0} \lambda}\left(\sum_{k=-\infty}^{k_{0}}\left(\sum_{j=-\infty}^{k-2} \mu\left(B_{j}\right)^{\frac{\alpha p}{n}}\left\|f \chi_{j}\right\|_{L^{q_{1}(\mu)}}^{p} W(j, k)\right)\right)^{\frac{1}{p}} \\
& \leq C\|b\|_{L i p_{\beta, \mu}} \sup _{k_{0} \in \mathbb{Z}} 2^{-k_{0} \lambda}\left(\sum_{j=-\infty}^{k_{0}} \mu\left(B_{j}\right)^{\frac{\alpha p}{n}}\left\|f \chi_{j}\right\|_{L^{q_{1}(\mu)}}^{p}\left(\sum_{k=j+2}^{\infty} W(j, k)\right)\right)^{\frac{1}{p}} \\
& =C\|b\|_{L i p_{\beta, \mu}} \sup _{k_{0} \in \mathbb{Z}} 2^{-k_{0} \lambda}\left(\sum_{j=-\infty}^{k_{0}} \mu\left(B_{j}\right)^{\frac{\alpha p}{n}}\left\|f \chi_{j}\right\|_{L^{q_{1}(\mu)}}^{p}\right)^{\frac{1}{p}} \\
& =C\|b\|_{L i p_{\beta, \mu}}\|f\|_{M K_{p, q_{1}}^{\alpha, \lambda}(\mu, \mu)} .
\end{aligned}
$$

For $I_{3}$, by analogy to the estimates of $I_{1}$, we find, for $j \geq k+2$,

$$
D_{1}(j, k) \leq C\|b\|_{L i p_{\beta, \mu}}(j-k) 2^{(k-j) n\left(\frac{\delta}{q_{1}}-\frac{\beta}{n}\right)}\left\|f \chi_{j}\right\|_{L^{q_{1}}(\mu)},
$$

and

$$
D_{2}(j, k) \leq C\|b\|_{L i p_{\beta, \mu}} 2^{(k-j) n \frac{1}{q_{2}}}\left\|f \chi_{j}\right\|_{L^{q_{1}}(\mu)} .
$$

Combining the estimates for $D_{1}(j, k)$ and $D_{2}(j, k)$, we obtain that if $j \geq k+2$, then

$$
\begin{aligned}
& \left\|\left([b, T] f \chi_{j}\right) \chi_{k}\right\|_{L^{q_{2}\left(\mu^{1-q_{2}}\right)}} \\
\leq & C\|b\|_{L_{p_{\beta}, \mu}}(j-k) 2^{(k-j) n\left(\frac{\delta}{q_{1}}-\frac{\beta}{n}\right)}\left\|f \chi_{j}\right\|_{L^{q_{1}}(\mu)}+C\|b\|_{L i p_{\beta, \mu}} 2^{(k-j) n \frac{1}{q_{2}}}\left\|f \chi_{j}\right\|_{L^{q_{1}}(\mu)} \\
\leq & C\|b\|_{L_{p_{\beta, \mu}}}(j-k) 2^{(k-j) n\left(\frac{\delta}{q_{1}}-\frac{\beta}{n}\right)}\left\|f \chi_{j}\right\|_{L^{q_{1}}(\mu)} .
\end{aligned}
$$

So we have

$$
\begin{aligned}
I_{3} \leq C & \sup _{k_{0} \in \mathbb{Z}} 2^{-k_{0} \lambda}\left(\sum_{k=-\infty}^{k_{0}} \mu\left(B_{k}\right)^{\alpha p / n}\right. \\
& \left.\times\left(\sum_{j=k+2}^{\infty}\|b\|_{L i p_{\beta, \mu}}(j-k) 2^{(k-j) n\left(\frac{\delta}{q_{1}}-\frac{\beta}{n}\right)}\left\|f \chi_{j}\right\|_{L^{q_{1}}(\mu)}\right)^{p}\right)^{\frac{1}{p}} \\
=C & \|b\|_{L i p_{\beta, \mu}} \sup _{k_{0} \in \mathbb{Z}} 2^{-k_{0} \lambda}\left(\sum _ { k = - \infty } ^ { k _ { 0 } } \left(\sum_{j=k+2}^{\infty} \mu\left(B_{j}\right)^{\frac{\alpha}{n}}\right.\right. \\
& \left.\left.\times\left(\frac{\mu\left(B_{k}\right)}{\mu\left(B_{j}\right)}\right)^{\frac{\alpha}{n}}(j-k) 2^{(k-j) n\left(\frac{\delta}{q_{1}}-\frac{\beta}{n}\right)}\left\|f \chi_{j}\right\|_{L^{q_{1}}(\mu)}\right)^{p}\right)^{\frac{1}{p}} .
\end{aligned}
$$

Let $V(j, k)=(j-k) 2^{(k-j) \delta\left(\alpha+n / \widetilde{q}_{2}\right)}$ and $N(j, k)=\left(\frac{\mu\left(B_{k}\right)}{\mu\left(B_{j}\right)}\right)^{\frac{\alpha}{n}}(j-k) 2^{(k-j) n\left(\frac{\delta}{q_{1}}-\frac{\beta}{n}\right)}$. 
If $\alpha \geq 0$, then by Lemma 2.1,

$$
\begin{aligned}
N(j, k) & \leq C 2^{(k-j) \delta \alpha}(j-k) 2^{(k-j) n\left(\frac{\delta}{q_{1}}-\frac{\beta}{n}\right)} \\
& =C(j-k) 2^{(k-j) \delta\left[\alpha+n\left(\frac{1}{q_{1}}-\frac{\beta}{n \delta}\right)\right]} \\
& \leq C(j-k) 2^{(k-j) \delta\left(\alpha+\frac{n}{q_{2}}\right)} \\
& =C V(j, k) .
\end{aligned}
$$

If $\alpha<0$, then by Lemma 2.1,

$$
\begin{aligned}
N(j, k) & \leq C 2^{(k-j) \alpha}(j-k) 2^{(k-j) n\left(\frac{\delta}{q_{1}}-\frac{\beta}{n}\right)} \\
& =C(j-k) 2^{(k-j)\left[\alpha+n\left(\frac{\delta}{q_{1}}-\frac{\beta}{n}\right)\right]} \\
& \leq C(j-k) 2^{(k-j)\left(\alpha+\frac{n}{q_{2}}\right)} \\
& \leq C V(j, k) .
\end{aligned}
$$

Thus, in both cases $\alpha \geq 0$ and $\alpha<0$, we have

$$
\begin{aligned}
I_{3} \leq & C\|b\|_{L i p_{\beta, \mu}} \sup _{k_{0} \in \mathbb{Z}} 2^{-k_{0} \lambda}\left(\sum_{k=-\infty}^{k_{0}}\left(\sum_{j=k+2}^{\infty} V(j, k) \mu\left(B_{j}\right)^{\alpha / n}\left\|f \chi_{j}\right\|_{L^{q_{1}}(\mu)}\right)^{p}\right)^{\frac{1}{p}} \\
\leq & C\|b\|_{L i p_{\beta, \mu}}\left(\sup _{k_{0} \in \mathbb{Z}} 2^{-k_{0} \lambda}\left(\sum_{k=-\infty}^{k_{0}}\left(\sum_{j=k+2}^{k_{0}} V(j, k) \mu\left(B_{j}\right)^{\alpha / n}\left\|f \chi_{j}\right\|_{L^{q_{1}}(\mu)}\right)^{p}\right)^{\frac{1}{p}}\right. \\
& \left.+\sup _{k_{0} \in \mathbb{Z}} 2^{-k_{0} \lambda}\left(\sum_{k=-\infty}^{k_{0}}\left(\sum_{j=k_{0}+1}^{\infty} V(j, k) \mu\left(B_{j}\right)^{\alpha / n}\left\|f \chi_{j}\right\|_{L^{q_{1}}(\mu)}\right)^{p}\right)^{\frac{1}{p}}\right) \\
:= & C\|b\|_{L i p_{\beta, \mu}}\left(I_{31}+I_{32}\right) .
\end{aligned}
$$

If $0<p \leq 1$, then it follows from $\alpha>\lambda / \delta-n / \widetilde{q_{2}} \geq-n / \widetilde{q_{2}}$ that

$$
\begin{aligned}
I_{31} & \leq \sup _{k_{0} \in \mathbb{Z}} 2^{-k_{0} \lambda}\left(\sum_{k=-\infty}^{k_{0}} \sum_{j=k+2}^{k_{0}} V(j, k)^{p} \mu\left(B_{j}\right)^{\alpha p / n}\left\|f \chi_{j}\right\|_{L^{q_{1}(\mu)}}^{p}\right)^{\frac{1}{p}} \\
& =\sup _{k_{0} \in \mathbb{Z}} 2^{-k_{0} \lambda}\left(\sum_{j=-\infty}^{k_{0}} \mu\left(B_{j}\right)^{\alpha p / n}\left\|f \chi_{j}\right\|_{L^{q_{1}}(\mu)}^{p}\left(\sum_{k=-\infty}^{j-2} V(j, k)^{p}\right)\right)^{\frac{1}{p}} \\
& =C \sup _{k_{0} \in \mathbb{Z}} 2^{-k_{0} \lambda}\left(\sum_{j=-\infty}^{k_{0}} \mu\left(B_{j}\right)^{\alpha p / n}\left\|f \chi_{j}\right\|_{L^{q_{1}}(\mu)}^{p}\right)^{\frac{1}{p}} \\
& =C\|f\|_{M K_{p, q_{1}}^{\alpha, \lambda}(\mu, \mu)^{.}}
\end{aligned}
$$


When $1<p<\infty$, by Hölder's inequality and $\alpha>\lambda / \delta-n / \widetilde{q_{2}} \geq-n / \widetilde{q_{2}}$, we have

$$
\begin{aligned}
I_{31} & \leq \sup _{k_{0} \in \mathbb{Z}} 2^{-k_{0} \lambda}\left(\sum_{k=-\infty}^{k_{0}}\left(\sum_{j=k+2}^{k_{0}} V(j, k)\right)^{\frac{p}{p^{\prime}}}\left(\sum_{j=k+2}^{k_{0}} \mu\left(B_{j}\right)^{\alpha p / n}\left\|f \chi_{j}\right\|_{L^{q_{1}(\mu)}}^{p} V(j, k)\right)\right)^{\frac{1}{p}} \\
& \leq C \sup _{k_{0} \in \mathbb{Z}} 2^{-k_{0} \lambda}\left(\sum_{k=-\infty}^{k_{0}} \sum_{j=k+2}^{k_{0}} \mu\left(B_{j}\right)^{\alpha p / n}\left\|f \chi_{j}\right\|_{L^{q_{1}(\mu)}}^{p} V(j, k)\right)^{\frac{1}{p}} \\
& =C \sup _{k_{0} \in \mathbb{Z}} 2^{-k_{0} \lambda}\left(\sum_{j=-\infty}^{k_{0}} \mu\left(B_{j}\right)^{\alpha p / n}\left\|f \chi_{j}\right\|_{L^{q_{1}}(\mu)}^{p}\left(\sum_{k=-\infty}^{j-2} V(j, k)\right)\right)^{\frac{1}{p}} \\
& =C \sup _{k_{0} \in \mathbb{Z}} 2^{-k_{0} \lambda}\left(\sum_{j=-\infty}^{k_{0}} \mu\left(B_{j}\right)^{\alpha p / n}\left\|f \chi_{j}\right\|_{L^{q_{1}}(\mu)}^{p}\right)^{\frac{1}{p}} \\
& =C\|f\|_{M K_{p, q_{1}}^{\alpha, \lambda}(\mu, \mu)}
\end{aligned}
$$

Thus, in both cases $0<p \leq 1$ and $1<p<\infty$, we have

$$
I_{31} \leq C\|f\|_{M K_{p, q_{1}}^{\alpha, \lambda}(\mu, \mu)} .
$$

For all $0<p<\infty$, it follows from $\alpha>\lambda / \delta-n / \widetilde{q_{2}}$ that

$$
\begin{aligned}
I_{32} \leq \sup _{k_{0} \in \mathbb{Z}} 2^{-k_{0} \lambda}\left(\sum _ { k = - \infty } ^ { k _ { 0 } } \left(\sum_{j=k_{0}+1}^{\infty} V(j, k) 2^{j \lambda} 2^{-j \lambda}\right.\right. \\
\left.\left.\quad \times\left(\sum_{l=-\infty}^{j} \mu\left(B_{l}\right)^{\alpha p / n}\left\|f \chi_{l}\right\|_{L^{q_{1}}(\mu)}^{p}\right)^{1 / p}\right)^{p}\right)^{\frac{1}{p}} \\
\leq\|f\|_{M K_{p, q_{1}}^{\alpha, \lambda}(\mu, \mu)} \sup _{k_{0} \in \mathbb{Z}} 2^{-k_{0} \lambda}\left(\sum_{k=-\infty}^{k_{0}}\left(\sum_{j=k_{0}+1}^{\infty} V(j, k) 2^{j \lambda}\right)^{p}\right)^{\frac{1}{p}} \\
=\|f\|_{M K_{p, q_{1}}^{\alpha, \lambda}(\mu, \mu)} \sup _{k_{0} \in \mathbb{Z}}\left(\sum_{k=-\infty}^{k_{0}} 2^{\left(k-k_{0}\right) \lambda p}\left(\sum_{j=k_{0}+1}^{\infty} V(j, k) 2^{(j-k) \lambda}\right)^{p}\right)^{\frac{1}{p}} \\
\leq\|f\|_{M K_{p, q_{1}}^{\alpha, \lambda}(\mu, \mu)} \sup _{k_{0} \in \mathbb{Z}}\left(\sum_{k=-\infty}^{k_{0}} 2^{\left(k-k_{0}\right) \lambda p}\left(\sum_{j=k_{0}}^{\infty}(j-k) 2^{(k-j) \delta\left(\alpha+n / \widetilde{q_{2}}-\lambda / \delta\right)}\right)^{p}\right)^{\frac{1}{p}} \\
\leq C\|f\|_{M K_{p, q_{1}}^{\alpha, \lambda}(\mu, \mu)} \sup _{k_{0} \in \mathbb{Z}}\left(\sum_{k=-\infty}^{k_{0}}\left(k_{0}-k\right)^{p} 2^{\left(k-k_{0}\right) \delta\left(\alpha+n / \widetilde{q_{2}}\right) p}\right)^{1 / p} \\
=C\|f\|_{M K_{p, q_{1}}^{\alpha, \lambda}(\mu, \mu)} .
\end{aligned}
$$

Thus,

$$
I_{3} \leq C\|b\|_{L_{i p_{\beta, \mu}}}\|f\|_{M K_{p, q_{1}}^{\alpha, \lambda}(\mu, \mu)} .
$$

Combining the estimates for $I_{1}, I_{2}$ and $I_{3}$, we complete the proof of Theorem 2.1. 


\section{ACKNOWLEDGMENTS}

The authors thank the referees for their careful reading of the manuscript and insightful comments.

\section{REFERENCES}

1. R. Coifman, R. Rochberg and G. Weiss, Factorization theorems for Hardy spaces in several variables, Ann. Math., 103 (1976), 611-635.

2. J. García-Cuerva, Weighted $H^{p}$ space, Dissert. Math., 162 (1979).

3. B. Hu and J. J. Gu, Necessary and sufficient conditions for boundedness of some commutators with weighted Lipschitz functions, J. Math. Appl., 340 (2008), 598-605.

4. S. Janson, Mean oscillation and commutators of singular integral operators, Ark. Math., 16 (1978), 263-270.

5. J. L. Journé, Calderón-Zygmund operators, pseudo-differential operators and the Cauchy integral of Calderón, Lecture Notes in Math., 994 (1983), 1-127.

6. Y. Lin, Strongly singular Calderón-Zygmund operator and commutator on Morrey type spaces, Acta Math. Sinica, 23 (2007), 2097-2110.

7. Y. Lin, P. Liu and Z. G. Liu, Weighted Lipschitz estimates for commutators of singular integrals with rough kernels, Communications in Math. Analysis, 7 (2009), 73-80.

8. Y. Lin, Z. G. Liu and G. X. Pan, Weighted Lipschitz estimates for commutators of fractional integrals with homogeneous kernels, Taiwanese J. Math., 15 (2011), 2689-2700.

9. Y. Lin, Z. G. Liu and M. M. Song, Lipschitz estimates for commutators of singular integral operators on weighted Herz spaces, Jordan Journal of Mathematics and Statistics, 3 (2010), 53-64.

10. S. Z. Lu and D. C. Yang, Boundedness of commutators on Hardy type spaces, Science in China (Ser A), 45 (2002), 984-997.

11. M. Paluszyński, Characterization of the Besov spaces via the commutator operator of Coifman, Rochberg and Weiss, Indiana Univ. Math. J., 44 (1995), 1-17.

12. F. Soria and G. Weiss, A remark on singular integrals and power weights, Indiana Univ. Math., 43 (1994), 187-204.

13. E. M. Stein, Note on singular integrals, Proc. Amer. Soc. Math., 8 (1957), 250-254.

Yan Lin, Zongguang Liu and $\mathrm{Na} \mathrm{Xu}$

School of Sciences

China University of Mining and Technology

Beijing 100083

P. R. China

E-mail: linyan@cumtb.edu.cn liuzg@cumtb.edu.cn xuna999@163.com 